\title{
Measurement-based synthesis of multiqubit entangled states in superconducting cavity QED
}

\author{
Ferdinand Helmer and Florian Marquardt \\ Department of Physics, Center for NanoScience, and Arnold Sommerfeld Center for Theoretical Physics, \\ Ludwig-Maximilians-Universität, Theresienstrasse 37, D-80333 Munich, Germany
}

(Received 4 February 2009; published 20 May 2009)

\begin{abstract}
Entangled multiqubit states may be generated through a dispersive collective quantum nondemolition measurement of superconducting qubits coupled to a microwave transmission line resonator. Using the quantum trajectory approach, we analyze the stochastic measurement traces that would be observed in experiments. We illustrate the synthesis of three-qubit $W$ and Greenberger-Horne-Zeilinger states, and we analyze how the fidelity and the entanglement evolve in time during the measurement. We discuss the influence of decoherence and relaxation, as well as of imperfect control over experimental parameters. We show that the desired states can be generated on time scales much faster than the qubit decoherence rates.
\end{abstract}

DOI: 10.1103/PhysRevA.79.052328 PACS number(s): 03.67.Bg, 03.65.Ud, 42.50.Pq, 42.50.Dv

\section{INTRODUCTION}

The realization of quantum-optical concepts in condensed matter systems has led to remarkable progress during the past few years. One of the prime examples is the study of quantum electrodynamics (QED) in superconducting circuits. Earlier suggestions to implement the JaynesCummings model in the solid state [1-3] were followed by a proposal [4] to employ on-chip microwave resonators and couple them to artificial atoms in the form of superconducting qubits. This seminal idea was soon thereafter realized experimentally [5], creating a solid-state analog of conventional optical cavity QED [6]. The tight confinement of the field mode and the large electric dipole moment of the "atom" yield extraordinary coupling strengths. As a result, these highly tunable systems have been employed to demonstrate experimentally a variety of achievements, including: the Jaynes-Cummings model in the strong-coupling regime $[5,7,8]$, Rabi and Ramsey oscillations and dispersive qubit readout $[9,10]$, generation of single photons [11] and Fock states $[12,13]$, cavity-mediated coupling of two qubits $[14,15]$, setups with three qubits [16], Berry's phase [17], and measurement of the photon number distribution [18].

The strong coupling makes dispersive quantum nondemolition (QND) readout possible, both for qubit states and for detecting single photons [19]. QND measurements are ideal projective measurements that reproduce their outcome when repeated $[20,21]$. Any QND measurement may be applied to (probabilistically) generate states. In particular, having several qubits inside a common cavity (as realized in recent circuit QED experiments [14,15]; for a schematic setup see Fig. 1), one may produce entangled multiqubit states, even without employing directly any qubit-qubit coupling. In the context of circuit QED, this option has been investigated previously in a series of remarkable studies [22-24]. However, these consider primarily two qubits, with a recent work [23] discussing the extension to more qubits in general terms. The present paper aims to go beyond these studies in several aspects. First, we present necessary conditions for being able to generate arbitrary multiqubit states out of a given subspace of the total multiqubit Hilbert space, using only single qubit operations and subsequent collective mea- surement. Second, we carry out detailed quantum jump trajectory simulations also for the case of three qubits, where $W$ and Greenberger-Horne-Zeilinger (GHZ) states may be produced. We show how entanglement is generated in the course of the measurement process, paying attention to the effects of relaxation and decoherence. Moreover, we analyze how imprecise fine-tuning of experimental parameters would lead to a loss of entanglement after its initial transient generation. Finally, we comment on possible experimental realizations. Such a measurement-based scheme complements other approaches for entanglement generation in circuit QED [4,25-32], based on unitary dynamics, and may prove advantageous for some purposes since generation and measurement are combined into one step. It might also be used to generate entanglement between qubits in spatially separated cavities, without any direct interaction.

\section{MODEL}

We investigate a QND scheme utilizing the coupling of superconducting qubits to a bosonic field mode of a microwave resonator as examined in $[4,5]$. The presence of excitations in the qubits inside the cavity gives rise to a frequency shift of the cavity mode, which can be observed dispersively via the phase shift of a transmitted beam. In turn, the measurement backaction leads to a projection of the qubits on a state that depends on (a) the chosen set of couplings and (b) the initial (product) state the qubits are prepared in.

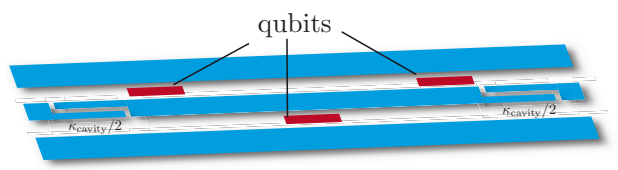

FIG. 1. (Color online) Schematic setup: three superconducting qubits (indicated by boxes between center conductor and ground planes) are coupled to a mode of a coplanar microwave resonator (consisting of a center conductor and two neighboring ground planes). The measurement of the phase shift of a transmitted microwave beam can be utilized to rapidly synthesize, e.g., maximally entangled multiqubit states such as GHZ and $W$ states. 
We consider a system of a driven cavity mode coupled to $N$ qubits,

$$
\begin{aligned}
\hat{H}= & \hbar \omega\left(\hat{a}^{\dagger} \hat{a}+\frac{1}{2}\right)+\sum_{i=1}^{N} \frac{\epsilon_{i}}{2}\left(\hat{\sigma}_{i}^{z}+1\right)+\sum_{i=1}^{N} g_{i}^{0}\left(\hat{\sigma}_{i}^{+} \hat{a}+\hat{\sigma}_{i}^{-} \hat{a}^{\dagger}\right) \\
& +\frac{\kappa_{\text {cavity }}}{2}\left(\varepsilon \hat{a}^{\dagger}+\hat{a} \varepsilon^{*}\right)+\hat{H}_{\text {decay }},
\end{aligned}
$$

a model commonly known as the Tavis-Cummings model which has been recently realized experimentally for $N=2$ $[14,15]$ and $N=3$ [16]. The first term of this Hamiltonian describes the cavity mode with a frequency $\omega$, the second describes all qubit energies, the third term realizes the Jaynes-Cummings coupling for each qubit to the cavity with bare coupling constants $g_{i}^{0}$, while the last term describes the driving of the cavity with the readout microwave tone which will yield $|\varepsilon|^{2}$ photons in the resonator on average ( $\kappa_{\text {cavity }}$ is the intensity decay rate for the cavity).

In the limit where all the qubits are strongly detuned from the cavity, it is well known [4] that the qubits impart a statedependent phase shift on the cavity mode and the effective Hamiltonian can be written as

$$
\begin{aligned}
\hat{H}= & \hbar \omega\left(\hat{n}+\frac{1}{2}\right)+\sum_{i=1}^{N} \frac{\epsilon_{i}}{2}\left(\hat{\sigma}_{i}^{z}+1\right)+\sum_{i=1}^{N} \frac{\left(g_{i}^{0}\right)^{2}}{\Delta_{i}} \frac{\left(\hat{\sigma}_{i}^{z}+1\right)}{2} \hat{n} \\
& +\frac{\kappa_{\text {cavity }}}{2}\left(\varepsilon \hat{a}^{\dagger}+\hat{a} \varepsilon^{*}\right)+\hat{H}_{\text {decay }} \\
= & \hbar\left[\omega+\sum_{i=1}^{N} \frac{\left(g_{i}^{0}\right)^{2}}{\Delta_{i}} \frac{\left(\hat{\sigma}_{i}^{z}+1\right)}{2}\right]\left(\hat{n}+\frac{1}{2}\right)+\sum_{i=1}^{N} \frac{\epsilon_{i}}{2}\left(\hat{\sigma}_{i}^{z}+1\right) \\
& +\frac{\kappa_{\text {cavity }}}{2}\left(\varepsilon \hat{a}^{\dagger}+\hat{a} \varepsilon^{*}\right)+\hat{H}_{\text {decay }} .
\end{aligned}
$$

Here, $\hat{n}=\hat{a}^{\dagger} \hat{a}$ denotes the number operator of the cavity mode. Hamiltonian (1) also induces an effective flip-flop interaction $[4,33]$ of strength $J_{\alpha \beta}=g_{\alpha} g_{\beta}\left(\Delta_{\alpha}+\Delta_{\beta}\right) /\left(2 \Delta_{\alpha} \Delta_{\beta}\right)$ between each pair of qubits $(\alpha, \beta)$ in the same cavity (for couplings $g_{\alpha(\beta)}$ and detunings from the cavity $\Delta_{\alpha(\beta)}$, in the dispersive limit $|g| \ll|\Delta|)$ :

$$
\hat{H}_{\alpha \beta}^{\text {flip-flop }}=J_{\alpha \beta}\left(\hat{\sigma}_{\alpha}^{+} \hat{\sigma}_{\beta}^{-}+\text {H.c. }\right) .
$$

When simulating master Eq. (11) to be derived from Hamiltonian (2), we neglect this interaction for several reasons. (i) In a concrete experiment the qubit energies could always be chosen very differently such that this unwanted interaction does not play a role since the qubits are nonresonant. (ii) As we will argue later in more detail, the measurement rate $\bar{\Gamma}$ is usually much larger than $J_{\alpha \beta}$, thus making the effects of the interaction negligibly small even when the qubits are in resonance with each other. (iii) We note that all the states we consider as examples are eigenstates of the flip-flop interaction [Eq. (3)]. Therefore, even if the qubits are chosen to be in resonance (as is ultimately assumed in our simulations), the interaction will not have any important effect on the dynamics besides trivial phases between subspaces that are rendered mutually incoherent by the measurement anyway. Thus, we will neglect the flip-flop interaction.

\section{NECESSARY CONDITIONS FOR THE GENERATION OF GIVEN TARGET STATES}

The protocol we are envisaging is to first produce a product state, using local operations on the individual qubits, and then to project on an entangled state by measurement. This scheme will be successful with a certain probability. Our aim in the present section is to briefly discuss the necessary conditions that must be met to be able to generate a given class of entangled states. It goes without saying that once an entangled state has been reached, one may then apply further local operations to reach a corresponding subspace of the full multiqubit Hilbert space.

Let us first fix notation. The coupling strengths $g_{i}$ determine the phase shifts induced by the individual qubits,

$$
g_{i} \equiv \frac{\left(g_{i}^{0}\right)^{2}}{\Delta_{i}},
$$

and for convenience we will collect them into the coupling vector

$$
\vec{G} \equiv\left(\frac{g_{i}}{\bar{g}}\right), \quad i=1, \ldots, N,
$$

where the overall strength $\bar{g}$ just determines the measurement time scale but does not affect the reachable states.

Using qubit excitation operators $\hat{n}_{i} \equiv \frac{\left(\hat{\sigma}_{i}^{7}+1\right)}{2}$, we define the measurement operator $\hat{N}$ as

$$
\hat{N} \equiv \sum_{i=1}^{N} \frac{g_{i}}{\bar{g}} \hat{n}_{i} .
$$

Note that even in current experiments, the $g_{i}$ are tunable in magnitude and sign simply by choosing the detuning $\Delta$ appropriately. The frequency shift imposed on the microwave cavity will then be

$$
\hat{\Phi}=\sum_{i=0}^{N} \hat{\phi}_{i}=\bar{g} \hat{N}=\sum_{i=1}^{N} g_{i} \hat{n}_{i},
$$

where $n_{i}$ is the excitation number of the $i$ th qubit.

The desired entangled state $|\Psi\rangle \equiv \sum_{j=1}^{2^{N}} \alpha_{j}\left|\varphi_{j}\right\rangle$ has complex amplitudes,

$$
\vec{\alpha} \equiv\left(\alpha_{j}\right), \quad j=1, \ldots, 2^{N},
$$

in the energy eigenbasis of the qubits (a product basis that diagonalizes $\hat{n}_{i}$ ).

In the following we derive necessary conditions for being able to produce arbitrarily chosen states out of some $M$-dimensional Hilbert space that is spanned by a subset of $M$ basis states $\left|\varphi_{j}\right\rangle$. In order to generate a certain target state given by arbitrary $\vec{\alpha}$, we need to adjust the couplings such that all base kets with nonvanishing $\alpha_{j}$ yield the same phase shift. Assume that the amplitude vector of the target state has $M \in\left\{1, \ldots, 2^{N}\right\}$ nonzero entries $\alpha, j \in\left\{1, \ldots, 2^{N}\right\}$ where the 
corresponding indices can be written as a family $F_{\alpha}$ with $\operatorname{dim}\left(F_{\alpha}\right)=M$. Then the goal is to use the measurement to project the system onto the subspace given by $\operatorname{span}\left[\left\{\left|\varphi_{\mathrm{j}}\right\rangle \mid j\right.\right.$ $\left.\left.\in \mathrm{F}_{\alpha}\right\}\right]$.

In the simplest case this is directly possible by choosing (i) an appropriate initial product state of the qubits (to fix the amplitudes) and (ii) a suitable coupling vector (to project onto the correct subspace).

Choosing an arbitrary initial product state allows for the choice of $2 N$ complex amplitudes. Due to normalization of the $N$ single qubit states and an arbitrary global phase for each of those states, we essentially have $2 N$ real parameters to choose.

The amplitude vector of the target state will-up to a constant common factor due to the renormalization after projection-be determined by the amplitudes of this initial state. This suggests that, in general (i.e., for arbitrary target states), we can only aim at reaching states that satisfy

$$
2 M-2 \leq 2 N \text {. }
$$

Again, we had to subtract 2 to account for the irrelevant global phase and normalization.

Note that for the maximal value of $M=2^{N}$, the last inequality does not hold for any $N>1$ and we recover the fact that arbitrary states are in general not product states. Note that we have just found a necessary condition for constructing arbitrary states out of an $M$-dimensional subspace. When choosing particular states, e.g., trivially separable states, one may still be able to construct those even if they formally violate Eq. (9).

We now turn to the question when it is possible to choose the couplings such that the measurement cannot distinguish the components of the target state from each other. This requirement of equal phase shifts formally corresponds to a set of $M-1$ equations

$$
\left\langle\varphi_{i}|\hat{N}| \varphi_{i}\right\rangle=\left\langle\varphi_{j}|\hat{N}| \varphi_{j}\right\rangle
$$

where $i, j$ denote successive indices out of $F_{\alpha}$.

As tunable parameters to our disposal we effectively have $N-1$ couplings (discounting the overall strength $\bar{g}$ ) so this set of equations will in general be solvable as long as $M$ $\leq N$ is fulfilled.

As we will demonstrate below in several examples, some of the most interesting entangled states, such as $W$ and GHZ states for three qubits, can be synthesized by this scheme. Indeed, they have $M=N$ for the $W$ and $M=2$ for the $\mathrm{GHZ}$ states and thus satisfy the necessary conditions discussed in this section.

\section{STOCHASTIC MASTER EQUATION}

In this section we turn to the quantum trajectory approach known from quantum optics [21,34-45]. The stochastic master equation to be presented below allows us to model the backaction of the phase-shift measurement and to produce individual realizations of the measured phase-shift signal. Stochastic master equations of the kind used in the present analysis have been successfully employed to, e.g., model single qubits subject to a continuous measurement $[22,46,47]$ or to study qubit projection [48] or state reduction of bipartite systems [49]. Moreover, the same structure of a stochastic master equation, e.g., emerges when analyzing trapped ion motion cooling using continuous measurements and feedback [50].

In the case of a cavity mode that decays much faster both than the qubit decoherence rates $\left(\kappa_{\text {cavity }} \gg \gamma_{1}, \gamma_{\phi}\right)$ and the couplings to the cavity mode $\kappa_{\text {cavity }} \gg g_{i} \forall i \in\{0,1, \ldots, N\}$, it is possible to adiabatically eliminate the cavity mode from the system and find for the stochastic master equation (in the interaction picture) for the qubits alone,

$$
\begin{aligned}
\dot{\hat{\rho}}= & \sum_{i=0}^{N} \gamma_{1}\left(\hat{\sigma}_{i}^{-} \hat{\rho} \hat{\sigma}_{i}^{+}-\frac{1}{2} \hat{\sigma}_{i}^{+} \hat{\sigma}_{i}^{-} \hat{\rho}-\frac{1}{2} \hat{\rho} \hat{\sigma}_{i}^{+} \hat{\sigma}_{i}^{-}\right) \\
& +\sum_{i=0}^{N} \gamma^{\varphi}\left[2 \hat{P}_{i} \hat{\rho} \hat{P}_{i}-\hat{P}_{i} \hat{\rho}-\hat{\rho}_{i}\right]-2 \bar{\Gamma}[\hat{N},[\hat{N}, \hat{\rho}]] \\
& -\sqrt{4 \bar{\Gamma}}[\hat{N} \hat{\rho}+\hat{\rho} \hat{N}-2 \hat{\rho}\langle\hat{N}\rangle(t)] \xi(t) .
\end{aligned}
$$

Here $\bar{\Gamma} \equiv \frac{\bar{g}^{2}|\varepsilon|^{2}}{\kappa_{\text {cavity }}}$ is the measurement rate, $\gamma_{1}$ and $\gamma_{\varphi}$ are the qubit relaxation and dephasing rates, $\hat{P}_{i}$ is the projector onto the excited state of qubit $i$, and $|\varepsilon|^{2}$ is the average photon number circulating inside the cavity mode. See $[43,51]$ for a detailed derivation and [19] for our recent analysis of photon detection in circuit QED using the same approach. The stochastic master equation is conditioned on the measured signal,

$$
X(t) \equiv\langle\hat{N}\rangle(t)+\frac{1}{4} \sqrt{\frac{1}{\bar{\Gamma}}} \xi(t),
$$

where $\xi$ represents the fundamental, unavoidable vacuum noise [with $\left.\left\langle\xi(t) \xi\left(t^{\prime}\right)\right\rangle=\delta\left(t-t^{\prime}\right)\right]$.

The dephasing term $[\hat{N},[\hat{N}, \hat{\rho}]]$ destroys the coherence between different eigenstates of the operator $\hat{N}$, which is the unavoidable consequence of the QND measurement process. As such, this term, although formally analogous to dephasing terms appearing, among many other places, in the context of models of intrinsic decoherence (such as the one introduced by Milburn [52], where the number operator would be replaced by the Hamiltonian), models the measurementinduced dephasing due to the projective measurement of the electromagnetic field leaking out a cavity mode. To elaborate, in our model the concept of a (measurement) bath is a physically meaningful and necessary ingredient rather than a conceptual framework to derive the physics of decoherence. Only to the latter, intrinsic decoherence provides an alternative description.

Physically, $X(t)$ is the appropriate (suitably normalized) quadrature component of the electric field transmitted through the cavity, which is proportional to the phase shift that indicates the multiqubit state. Experimentally, this signal would be measured in a homodyne detection scheme. Note that, for a two-sided cavity, information is contained both in the transmitted and the reflected signal, and we have as- 
sumed that both parts of the signals are superimposed symmetrically to extract the maximum possible information content [19].

\section{EXAMPLES OF MEASUREMENT-GENERATED ENTANGLED STATES}

In this section we discuss the most relevant examples for the case of two and three qubits in the cavity. More precisely we will show that it is possible to generate Bell states, $W$ states, and GHZ states.

We will be able to observe that the measurement indeed first drives the system to one of its attractor solutions (among them the desired state) which are then stabilized by the measurement. The attractor nature of the subspaces selected by the coupling vector $\vec{G}$ can be immediately understood from the structure of the stochastic master equation (11) by realizing that the stochastic term and the measurement-induced dephasing term both vanish if the density matrix is in the desired state. Only relaxation and dephasing can take the system out of this final state, and we will discuss their influence later.

\section{A. Quantitative characterization}

In order to characterize the time evolution during the measurement process, we have plotted several quantities. We plot the phase-shift signal $X(t)$ and the excitation number $n_{i}$ in each qubit as functions of time. To verify that we have indeed obtained the desired state, we will compute the state fidelity $\digamma$ between the density matrix from the simulation, $\rho_{\text {sim }}$, and the ideal state density matrix $\sigma$, according to $\digamma$ $\equiv \operatorname{tr}\left|\sqrt{\rho_{\text {sim }}} \sigma \sqrt{\rho_{\text {sim }}}\right|$. Finally, the two-qubit entanglement between two qubits $A$ and $B$ will be measured by the log negativity. Given the density matrix $\rho$ of the two qubits (after tracing out other qubits, if needed), this is defined as $E_{N}(\rho)$ $=\log _{2}\left\|\rho^{T_{A}}\right\|$. Here $\rho^{T_{A}}$ is the partial transpose with respect to qubit $A$, and $\|\mu\|=\operatorname{tr}\left[\sqrt{\mu^{\dagger} \mu}\right]$ is the trace norm.

Before discussing the individual examples, we briefly point out the general features. Looking at the results (e.g., in Fig. 2), we find that in those cases where we end up in the right state the fidelity as well as the log negativity are 1 . Furthermore, the state is stabilized by the measurement, meaning that, due to the absence of any nonvanishing terms in the master equation's right hand side, it is frozen. We observe that the state is generated on a time scale given by the measurement rate $\bar{\Gamma}^{-1}$.

While discussing the examples we will also analyze plots that show the probability density of various quantities evolving over time. This point merits a brief discussion. The time evolution of the distribution for any simple quantum mechanical observable can be immediately obtained from the time evolution of the average density matrix, i.e., from the standard, nonstochastic master equation. In that case, simulating a large number of stochastic trajectories and then averaging over the results would be unnecessarily cumbersome. However, that argument becomes void as soon as one considers signals that depend on the entire prehistory of the
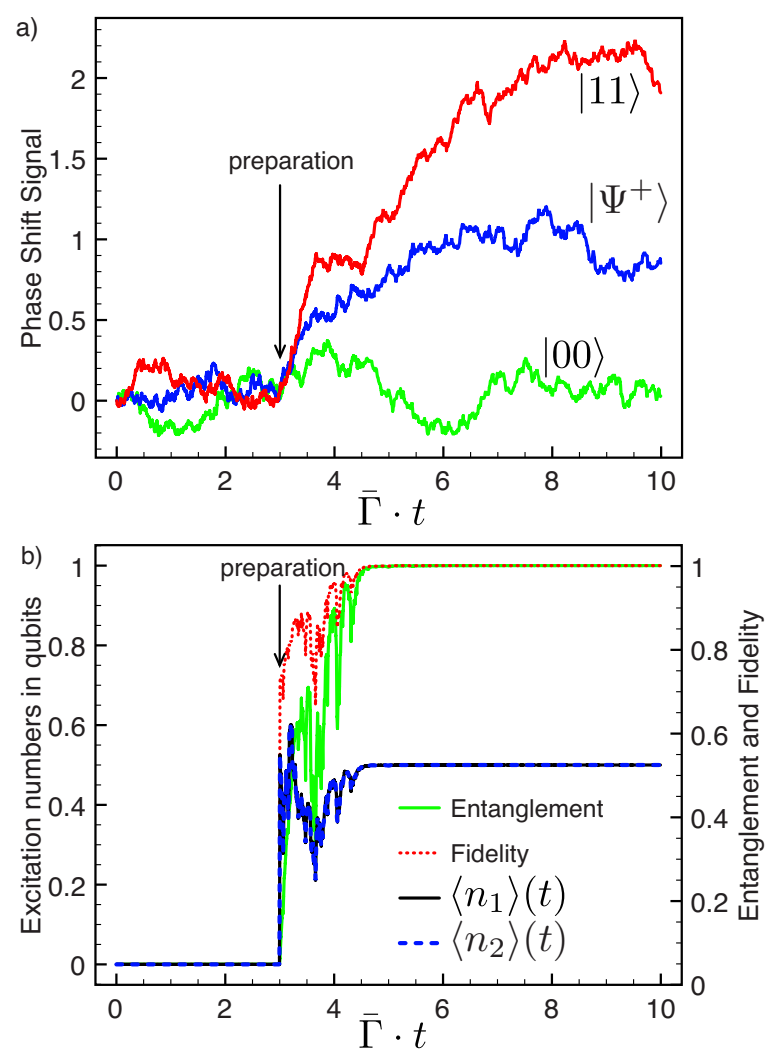

FIG. 2. (Color online) Generation of the Bell state $\left|\Psi^{+}\right\rangle=(|10\rangle$ $+|01\rangle) / \sqrt{2}$ (the two-qubit $W$ state): (a) quantum trajectories illustrating the different phase-shift signal traces $X(t)$. Three traces have been selected, corresponding to the possible outcomes of the measurement given the same input state $\left|\Psi_{0}\right\rangle$. At time $\bar{\Gamma} t_{0}=3$, Hadamard gates are applied to both qubits, starting from the ground state. As in every real measurement of field quadratures, the signal $X(t)$ is smoothed by doing a windowed average over a suitable time span, $\bar{\Gamma} \tau_{\text {avg }}=1.0$. Part (b) displays the excitation numbers, state synthesis fidelity and the entanglement (log negativity) for the one trajectory of plot (a) that ended up in the desired state.

trajectory. An important example is the time-averaged cumulative phase-shift signal,

$$
\bar{X}(t) \equiv \frac{1}{t} \int_{0}^{t} X\left(t^{\prime}\right) d t^{\prime}
$$

This quantity has the advantage of tending toward a welldefined limit in the course of a QND measurement, with the fluctuations around that limiting value decreasing such as $1 / \sqrt{t}$. It is not possible to obtain the distribution of $\bar{X}$ from the average density matrix $\rho$, and quantum jump trajectory simulations are needed.

Another example is represented by quantities that depend nonlinearly on the density matrix. In those cases, the average density matrix is irrelevant since obviously $\langle f(\rho)\rangle \neq f(\langle\rho\rangle)$ for a nonlinear function $f$. An important case is the entanglement measure $E_{N}$. In fact, the average density matrix is never entangled $\left[E_{N}(\langle\rho\rangle) \equiv 0\right]$ for our examples. Thus, it is indeed necessary to obtain $E_{N}$ for a large number of trajectories in 
order to discuss its statistical behavior and plot the probability density.

\section{B. Bell states for two qubits-no decoherence}

In the case of two qubits and vanishing decoherence rates $\gamma_{1}, \gamma_{\phi}=0$, the generation of Bell states is straightforward [23]. We imagine starting the experiment with all qubits in the ground state $|00\rangle$ and applying a Hadamard gate $\left(\pi / 2-\sigma_{x}\right.$ rotation $)$ at some time $t_{0}$, which leaves the system in the product state $\left|\Psi_{0}\right\rangle \equiv \Pi_{\otimes} \frac{1}{\sqrt{2}}(|0\rangle+|1\rangle)=\frac{1}{2}(|00\rangle+|01\rangle$ $+|10\rangle+|11\rangle)$. We want to generate the Bell state

$$
\left|\Psi^{+}\right\rangle \equiv \frac{1}{\sqrt{2}}(|01\rangle+|10\rangle)
$$

which is the two-qubit version of a $W$ state. Clearly the amplitude vector for this state is simply $\sqrt{2} \vec{\alpha}=(0,1,1,0)^{T}$, and the resulting equation (10) for the couplings is given by $g_{1}$ $=g_{2}$, thus $\vec{G}=(1,1)^{T}$. The desired state will be generated with a success rate $\eta$ given by

$$
\eta \equiv\left|\left\langle\Psi^{+} \mid \Psi_{0}\right\rangle\right|^{2}=\frac{1}{2}
$$

meaning that the experiment will in $50 \%$ of all runs end up in the correct state (as confirmed by observation of the correct phase shift).

Likewise, for the Bell state vector $\left|\Phi^{+}\right\rangle \equiv \frac{1}{\sqrt{2}}(|00\rangle+|11\rangle)$, we find for the amplitude vector $\sqrt{2} \vec{\alpha}=(1,0,0,1)^{T}$, and for the characteristic equation for the couplings $g_{1}=-g_{2}$, which is fulfilled by the choice of coupling vector $\vec{G}=(1,-1)^{T}$. Note that in principle $\left|\Phi^{+}\right\rangle$could also be generated by first producing $\left|\Psi^{+}\right\rangle$and then applying local unitary operations, and the same is true for the two other Bell states, $\left|\Psi^{-}\right\rangle$and $\left|\Phi^{-}\right\rangle$.

Individual traces and probability density time evolutions for various quantities are shown in Figs. 2 and 3, respectively, for the two types of Bell states discussed here.

\section{Three qubits—no decoherence}

\section{Generation of $W$ states}

Similarly, for three qubits, the generation of $W$ states is straightforward as well. We imagine starting the experiment with all qubits in the ground state $|0\rangle$, applying a Hadamard gate $\left(\pi / 2-\sigma_{x}\right.$ rotation) at some time $t_{0}$, leaving the system in the state

$$
\begin{aligned}
\left|\Psi_{0}\right\rangle \equiv & \prod_{\otimes} \frac{1}{\sqrt{2}}(|0\rangle+|1\rangle) \\
= & \frac{1}{\sqrt{8}}[|000\rangle+|001\rangle+|010\rangle+|011\rangle+|100\rangle+|101\rangle \\
& +|110\rangle+|111\rangle] .
\end{aligned}
$$

We aim to generate a $W$ state which for three qubits is given by
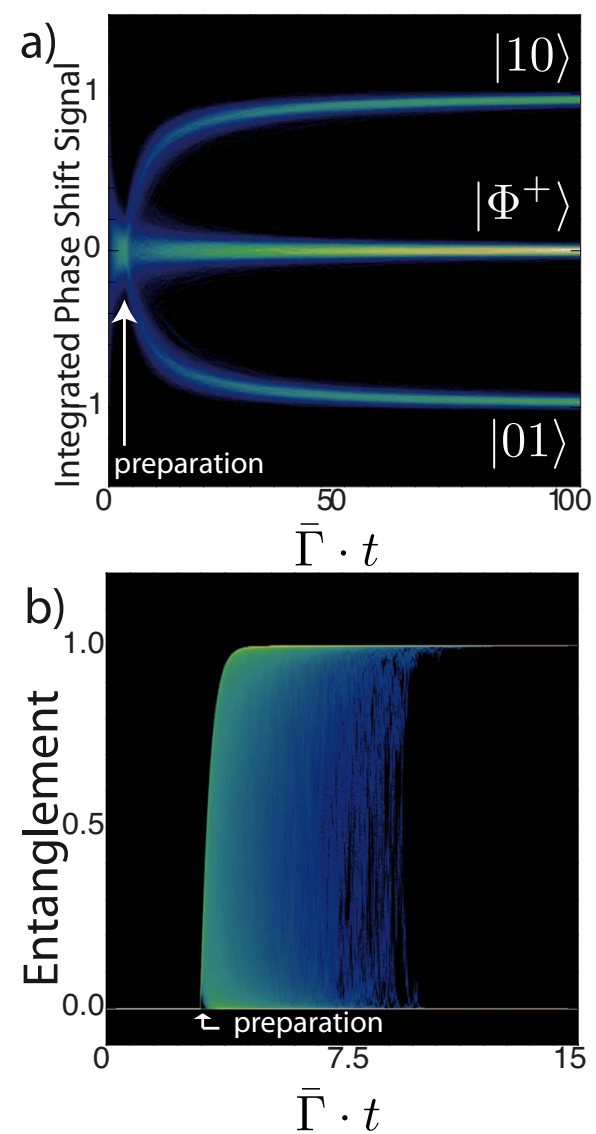

FIG. 3. (Color online) Generation of two-qubit Bell state $\left|\Phi^{+}\right\rangle$ $=(|00\rangle+|11\rangle) / \sqrt{2}$ : (a) Probability density of the integrated (cumulative) phase-shift signal $\bar{X}(t)=t^{-1} \int_{0}^{t} X\left(t^{\prime}\right) d t^{\prime}$ from 6000 runs of the simulation. At time $t_{0}$ Hadamard gates are applied to both qubits. Part (b) displays the probability density of the entanglement measure $E_{N}$, the log negativity. Note that neither of these plots can be obtained from the standard, nonstochastic master equation (see main text), i.e., quantum jump trajectory simulations are essential.

$$
|W\rangle \equiv \frac{1}{\sqrt{3}}(|001\rangle+|010\rangle+|100\rangle) .
$$

We find the corresponding amplitude vector $\sqrt{3} \vec{\alpha}$ $=(0,1,1,0,1,0,0,0)^{T}$, and the resulting equations for the couplings, $g_{1}=g_{2}=g_{3}$, solved by equal couplings to all qubits, $\vec{G}=(1,1,1)^{T}$. The $W$ state will be generated with a success rate $\eta$ given by

$$
\eta \equiv\left|\left\langle W \mid \Psi_{0}\right\rangle\right|^{2}=\frac{3}{8} .
$$

Note that with the same success rate the dual $W$ state,

$$
|\bar{W}\rangle \equiv \frac{1}{\sqrt{3}}(|011\rangle+|110\rangle+|101\rangle),
$$

is generated (see Fig. 4).

\section{Generation of GHZ states}

Extending the two-qubit scheme to three qubits, we find for the amplitude vector $\sqrt{3} \vec{\alpha}=(1,0,0,0,0,0,0,1)^{T}$ and for 

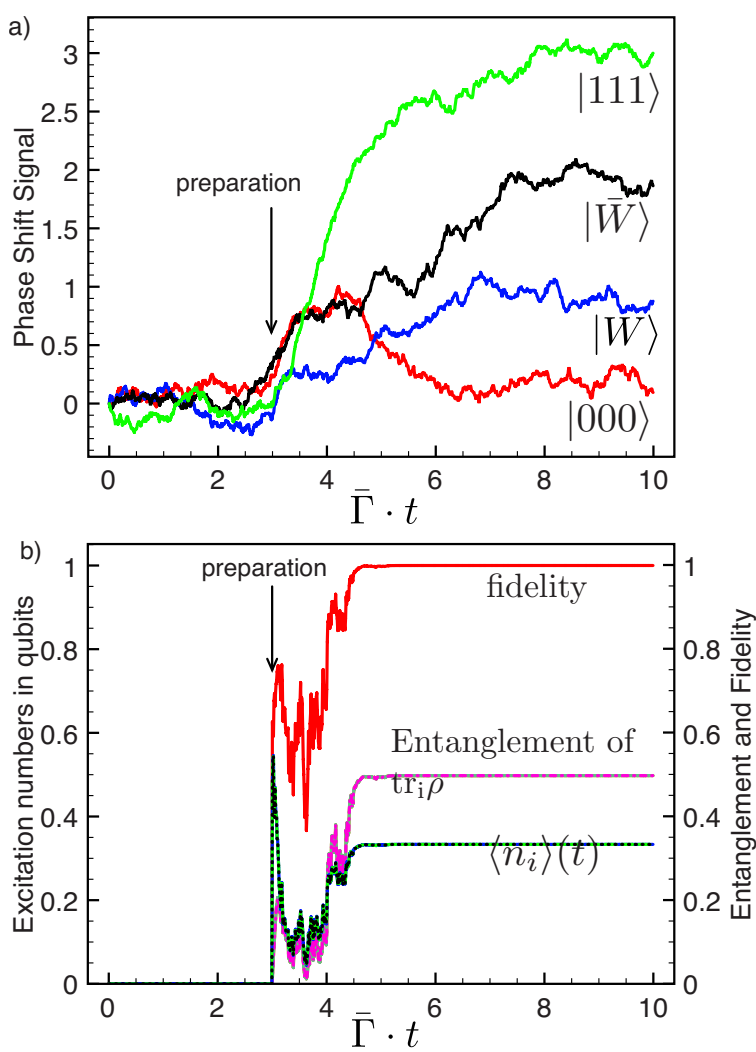

FIG. 4. (Color online) Generation of three-qubit $W$ states: (a) quantum trajectories for the different states that can arise from the given input state $\left|\Psi_{0}\right\rangle$. At time $\bar{\Gamma} t_{0}=3$, Hadamard gates are applied to all qubits. Windowed averaging is performed as in Fig. 2. Part (b) displays the excitation numbers, state synthesis fidelity and the log negativity for the one trajectory of plot (a) that ended up in the desired $W$ state. Here $\operatorname{tr}_{i} \rho$ denotes the partial trace over qubit number $i$, and the resulting pairwise entanglement happens to be the same for all choices of qubit pairs in this example. Note that in the target state all pairs of qubits are mutually entangled which is characteristic for the $W$ state and the reason for the robustness of its entanglement compared to the GHZ state.

the characteristic equation for the couplings in case of a desired GHZ state as the target state $|\mathrm{GHZ}\rangle \equiv \frac{1}{\sqrt{2}}(|000\rangle+|111\rangle)$,

$$
0=g_{1}+g_{2}+g_{3},
$$

which is fulfilled, for example, by the choice of coupling vector $\vec{G}=(1,-1 / 2,-1 / 2)^{T}$. The success rate is $\eta=\frac{1}{4}$. Again we plot phase-shift signal, excitation numbers, log negativity, and fidelity to illustrate the correctness of our considerations (see Fig. 5). Note that due to the unequal couplings, the qubit excitations and pairwise entanglement do depend on the qubit index, in contrast to all our previous examples, where the couplings had been equal in magnitude.

It is noteworthy that this three-qubit GHZ scheme yields a $75 \%$ chance of obtaining a Bell state between qubits 2 and 3 as a by-product. So this might in fact be also considered an even more efficient scheme to generate two-qubit Bell states than just with two qubits in the cavity.
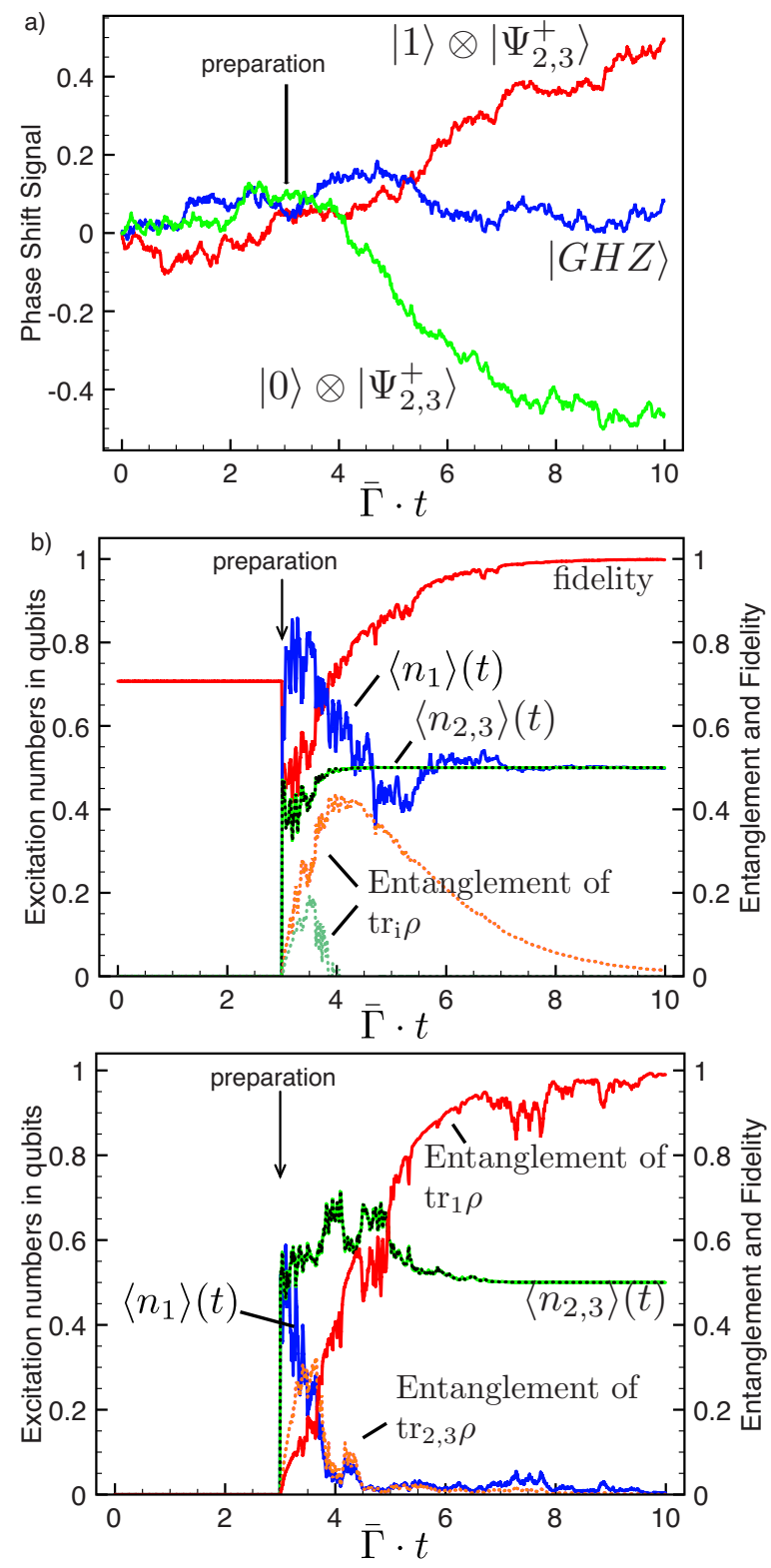

FIG. 5. (Color online) generation of three-qubit GHZ states: (a) quantum trajectories corresponding to the different states that can arise from the given input state. At time $t_{0}$, Hadamard gates are applied to both qubits. Note that among the unwanted outcomes there are two-qubit $\left|\Psi^{+}\right\rangle$Bell states. These are actually generated with a success rate of $\eta=3 / 4$ which is higher than in the original two-qubit scheme. Part (b) displays the excitation numbers, state synthesis fidelity and the log negativity for all pairs of qubits for the trace of part (a) that ended up in the desired GHZ state. Note that once the GHZ state is reached, all pairwise entanglement is lost. This is a typical feature of GHZ states, which contain only genuine three-particle entanglement. Part (c) shows the evolution for the particular trajectory that reaches the Bell state between qubits 2 and 3 , which can be generated very efficiently as a by-product using this three-qubit GHZ scheme.

\section{EFFECTS OF DECOHERENCE}

We include decoherence into our model by considering the stochastic master equation (11) with the Lindblad decay 


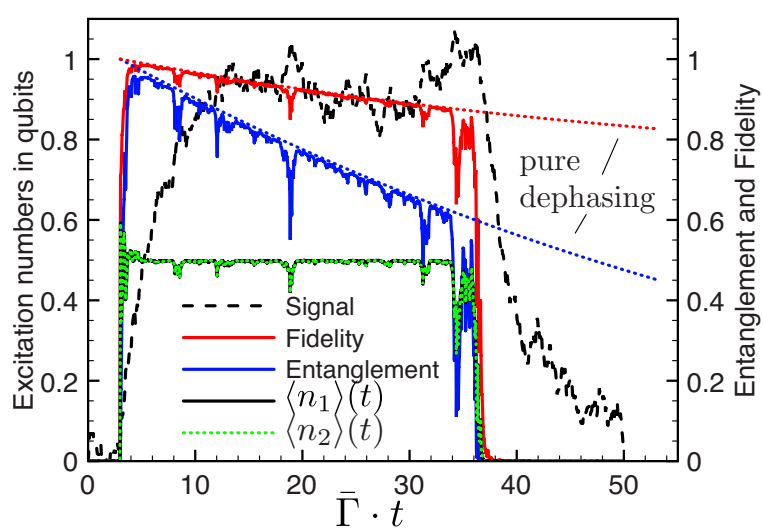

FIG. 6. (Color online) Effects of adding decoherence to the dynamics. The situation is identical to the simulation of Fig. 2, with the target state $\left|\Psi^{+}\right\rangle$, except for the added relaxation rate $\gamma_{1}$ $=0.01 \bar{\Gamma}$ and pure dephasing rate $\gamma_{\phi}=0.02 \Gamma$. We can observe that the subspace of choice is stabilized before the eventual decay due to relaxation. However, even before the sudden jump due to relaxation, one observes a slow decay of the fidelity and entanglement between the qubits, due to the pure dephasing rate $\gamma_{\phi}$ (dashed lines).

and dephasing rates now different from zero. Assuming equal rates for all the qubits, evidently entanglement will be on average destroyed on a time scale set by $T_{2}=\left(\gamma_{1} / 2\right.$ $\left.+\gamma_{\phi}\right)^{-1}$. When considering experimentally reachable parameters, which we will do further below, we will find that indeed the time needed to synthesize states is orders of magnitude shorter compared to $T_{2}$. It is thus clear that the simulation of the examples will look like the above with a weak decay of coherence superimposed on the trajectories.

In contrast to the decay due to decoherence, the decay due to relaxation (at a rate $\gamma_{1}$ ) is stochastic in the sense that it leads to sudden quantum jumps. This can be understood by considering that the phase-shift measurement stabilizes a certain subspace. Doing so, certain configurations of diagonal elements in the density matrix constitute attractors that compete with the exponential decay due to $\gamma_{1}$. More formally speaking, the master equation is unraveled with respect to the $\gamma_{1}$ process but is still an ensemble average description of the pure dephasing physics. The result is that the decay is stochastic when looking at single trajectories and the usual exponential $\gamma_{1}$ decay is recovered when averaging over many trajectories. Conversely, in a single trajectory the offdiagonal elements decay on a time scale set by $T_{2}$, showing the following behavior: as long as the relaxation jump process has not happened, one observes a decay solely due to pure dephasing (see Fig. 6). Once the relaxation process has happened, coherence and thus entanglement are also lost completely.

\section{Example-two-qubit Bell states including dissipation}

To demonstrate the influence of decoherence and relaxation, we repeat the example for a two-qubit Bell state, $\left|\Psi^{+}\right\rangle \equiv \frac{1}{\sqrt{2}}(|01\rangle+|10\rangle)$, assuming comparatively low values of $\bar{\Gamma} / \gamma_{1}=10$ and $\bar{\Gamma} / \gamma_{\phi}=20$ to illustrate the effects and make all
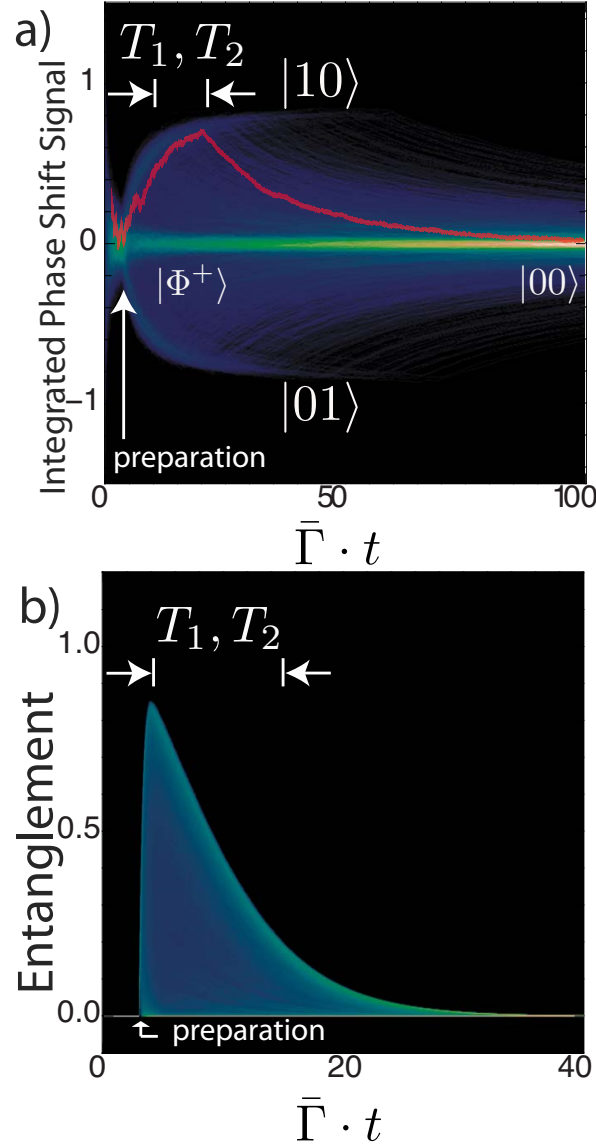

FIG. 7. (Color online) Effects of adding decoherence and relaxation to the creation of the Bell state $\left|\Phi^{+}\right\rangle \equiv \frac{1}{\sqrt{2}}(|00\rangle+|11\rangle)$. The situation is identical to the simulation of Fig. 3, except for the added relaxation rate $\gamma_{1}=0.1 \bar{\Gamma}$ and pure dephasing rate $\gamma_{\phi}=0.05 \Gamma$. Part (a) shows the probability density of the time-averaged (cumulative) phase-shift signal $\bar{X}(t)$ with an example trajectory superimposed. Note the buildup of finite probability at finite signal values, before relaxation back to zero phase shift, which represents the vacuum state $|00\rangle$ at long times. Part (b) shows the probability density of the entanglement ( $\log$ negativity). We can observe that the entanglement is lost on a time scale given by $T_{2}=\left(\gamma_{1} / 2+\gamma_{2}\right)^{-1}$. Note in particular the sharply defined, exponentially decaying envelope that defines a strict upper bound for the entanglement at any given time. This is due to the pure dephasing.

the dynamics visible. Experimental ratios would be at least about a factor 100 higher and thus the fidelity and lifetime are higher in experiment than they appear from the following simulations.

We have plotted the time evolution for the choice of couplings that leads to the creation of a two-qubit Bell state $\left|\Psi^{+}\right\rangle \equiv \frac{1}{\sqrt{2}}(|10\rangle+|01\rangle)$. The results are shown in Fig. 6, which should be compared against Fig. 2. Likewise, we have considered the probability density for the time-averaged phaseshift signal and the entanglement measure for the Bell state $\left|\Phi^{+}\right\rangle$; see Fig. 7. There, the strict upper envelope for the entanglement is particularly noteworthy, corresponding to the decay of coherence within the subspace selected by the measurement. 


\section{EFFECT OF IMPERFECTIONS DUE TO PARAMETER SPREAD}

In order to prepare states in this way experimentally, one faces the problem that it might not always be possible to fix important parameters perfectly. If the scheme one has in mind in turn relies on exact matching of parameters too much, one quickly ends up with a proposal that may be interesting but not very realistic. We therefore examine the effects on the fidelity and entanglement properties of this state synthesis scheme in the presence of small deviations in the couplings of the qubits to the cavity

$$
\vec{G}=\vec{G}_{0}+\left(\frac{\delta g_{i}}{\bar{g}}\right),
$$

where $\vec{G}_{0}$ is the ideal coupling vector from solving the characteristic equations (10) for the target state. $\delta g_{i} / \bar{g}$ are the deviations from that ideal coupling for each qubit. Without loss of generality we first look at the case where all couplings are equal to their ideal value, except one which differs by $\delta g / \bar{g}$. We focus on the stochastic term in the master equation [Eq. (11)] which is responsible for the projection onto a set of states, one of which is our target state. Let us rewrite this term a bit by inserting the definitions of $\bar{\Gamma}$ and $\hat{N}$ as follows:

$$
\begin{aligned}
& \dot{\hat{\rho}}_{\mathrm{st}}=-\sqrt{4 \bar{\Gamma}}[\hat{N} \hat{\rho}+\hat{\rho} \hat{N}-2 \hat{\rho}\langle\hat{N}\rangle(t)] \xi(t) \\
& =-\sqrt{4 \frac{\bar{g}^{2}|\alpha|^{2}}{\kappa_{\text {cavity }}}} \sum_{i=1}^{N} \frac{g_{i}}{\bar{g}}\left[\hat{n}_{i} \hat{\rho}+\hat{\rho} \hat{n}_{i}-2 \hat{\rho}\left\langle\hat{n}_{i}\right\rangle(t)\right] \xi(t) .
\end{aligned}
$$

From this form of Eq. (17), it is evident that due to the linearity in the couplings $g_{i}$, we can pull out all deviating couplings into separate terms which have the same form. This reads as

$$
\begin{aligned}
\dot{\hat{\rho}}= & -\sqrt{4 \bar{\Gamma}} \sum_{i=1}^{N} \frac{g_{i}^{(0)}}{\bar{g}}\left[\hat{n}_{i} \hat{\rho}+\hat{\rho} \hat{n}_{i}-2 \hat{\rho}\left\langle\hat{n}_{i}\right\rangle(t)\right] \xi(t) \\
& -\sqrt{4 \bar{\Gamma}} \sum_{i=1}^{N} \frac{\delta g_{i}}{\bar{g}}\left[\hat{n}_{i} \hat{\rho}+\hat{\rho} n_{i}-2 \hat{\rho}\left\langle\hat{n}_{i}\right\rangle(t)\right] \xi(t) \\
= & -\sqrt{4 \bar{\Gamma}}[\hat{N} \hat{\rho}+\hat{\rho} \hat{N}-2 \hat{\rho}\langle\hat{N}\rangle(t)] \xi(t) \\
& -\sum_{i=1}^{N} \sqrt{4 \delta \Gamma_{i}}\left[\hat{n}_{i} \hat{\rho}+\hat{\rho} \hat{n}_{i}-2 \hat{\rho}\left\langle\hat{n}_{i}\right\rangle(t)\right] \xi(t),
\end{aligned}
$$

which means that in addition to the ideal behavior captured by the first term, each individual qubit with deviating coupling will be projected on its ground or excited state on a time scale given by the inverse of the individual measurement rate $\delta \Gamma_{i} \equiv \frac{|\varepsilon|^{2} \delta g_{i}^{2}}{\kappa_{\text {cavity }}}$ (we have assumed positive $\delta g_{i}$ for simplicity; otherwise the signs in the last line would change for those qubits with $\left.\delta g_{i}<0\right)$. This has two consequences: The first consequence concerns the measured phase shift: Instead of being equal for all the base kets that form our target state, there will be deviations in the phase shift from base ket to base ket. This means that we will be able to observe the breakdown of the target state. Therefore, second, the lifetime

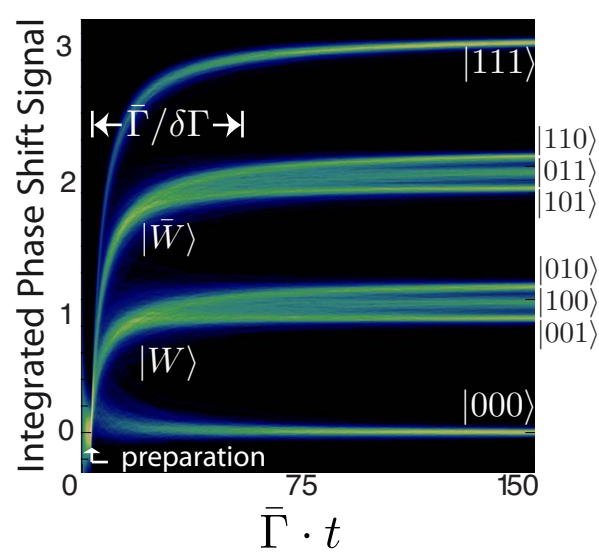

FIG. 8. (Color online) Probability density of the time-averaged cumulative (integrated) phase-shift signal $\bar{X}$ as an illustration of the effect of parameter spread in the couplings. Apart from the deviation in the couplings from the ideal values, the setup is identical to the example in which we aimed for a three-qubit $W$ state, as seen in Fig. 4. Hadamard gates are applied to all qubits at time $t_{0}=3 \bar{\Gamma}^{-1}$. During the following time interval of length $\bar{\Gamma}^{-1}$ all trajectories are projected onto the $W$ state, $|\bar{W}\rangle$, $|000\rangle$, or $|111\rangle$. Meanwhile the competing projection on the individual number states of the qubits becomes more pronounced and dominates the dynamics on a time scale $\delta \Gamma_{2}^{-1}=50 \bar{\Gamma}^{-1}$. This is exactly the time scale on which we can be sure to identify all the product base states by their phase-shift values individually.

of the desired entangled state will now also be limited by the inverse of the maximum of the individual measurement rates, in addition to the effects of decoherence. In other words, as soon as we have gained enough signal to noise ratio to discriminate the different base kets from each other (i.e., resolve the different corresponding phase shifts), our target state will be destroyed.

To illustrate this effect in a fairly drastic way, we choose an example of three qubits with a $W$ state as a target state and the coupling vector $\vec{G}=(1,1,1)^{T}+(\sqrt{2} / 10,0,-\sqrt{2} / 10)^{T}$. This yields an individual measurement rate for the second qubit of $\delta \Gamma_{2} / \bar{\Gamma}=1 / 50$. Therefore, we expect the target state and especially its entanglement properties to be destroyed on a time scale of 50 times the preparation time $\bar{\Gamma}^{-1}$. As we will argue in the following section, present experiments allow a ratio $\bar{\Gamma} / \gamma_{\text {decoh }}=O\left(10^{4}\right)$ which justifies to ignore decoherence for the moment. The resulting simulation beautifully confirms the expectations; see Fig. 8.

We conclude that in order to observe the full dynamics of the system one should strive for a regime where the condition

$$
\bar{\Gamma} \gg \delta \Gamma_{i}>\gamma_{1}, \gamma_{\phi} \quad \forall i \in 1, \ldots, N
$$

is met. In the next section we will show that this is indeed possible with present-day experimental parameters.

As a side remark we state that the situation of one coupling deviating from the others is in principle already found when synthesizing GHZ states for an odd number of qubits (e.g., three) as examined in the previous sections [see Fig. 
5(a)]. We had chosen a coupling vector $\vec{G}=(1,-1 / 2$, $-1 / 2)^{T}$. Here the larger magnitude of the coupling for the first qubit is responsible for the generation of two-qubit $W$ states. As a consequence, we can learn about the state of the first qubit while we can still not distinguish qubits 2 and 3 from each other. Following our previous reasoning in this section, we find that the state of qubit 2 should be discerned on a time scale set by $\left(\delta \Gamma_{2} / \bar{\Gamma}\right)^{-1}=4$ (in units of $\bar{\Gamma}^{-1}$ ), which matches the simulation results shown in the previous section [see Fig. 5(a)].

\section{POSSIBILITIES FOR EXPERIMENTAL REALIZATION}

Cavity QED setups in superconducting circuits $[5,8,53,54]$ have been used to implement ideas of quantum optics on the chip, and are considered a promising candidate for scalable, fault tolerant quantum computing (e.g., [55]). Proposals for generating and detecting nonclassical photon states exist or have been implemented [18,19,53,56,57].

These experiments realize a Jaynes-Cummings coupling between qubit and resonator of up to $2 \pi \times 100 \mathrm{MHz}$, resonators with frequencies of about $2 \pi \times 5 \mathrm{GHz}$, and a large range of resonator decay rates $\kappa$ between $10 \mathrm{kHz}$ and 100 MHz. Given this parameter space and assuming a bare qubit coupling of $g_{0} \approx 2 \pi \times 100 \mathrm{MHz}$, detunings in the $\mathrm{GHz}$ range, $|\epsilon|^{2} \approx 10$ photons in the readout cavity, and a qubit decay rate $\gamma_{1} \approx 0.6 \mathrm{MHz}$, it is easily possible to reach values of $\bar{\Gamma} / \kappa \approx 10^{4}$. This gives ample time for the state synthesis before decoherence starts playing a role.

Furthermore, couplings can be adjusted with enough accuracy such that the state generation is also not limited by this factor. We can examine the sensitivity of the ratio $\delta \Gamma / \bar{\Gamma}$ to small deviations in the parameters. From $\delta \Gamma \propto \delta g^{2}$ and $\delta g=\delta\left(g_{0}^{2} / \Delta\right)$, we find $\delta \Gamma / \bar{\Gamma}=\left[2 \delta g_{0} / g_{0}-\delta \Delta / \Delta\right]^{2}$. Assuming an uncertainty about the bare value of the coupling of the qubits to the cavity and an uncertainty about the qubit detuning of about $5 \%$ each we find that $\delta \Gamma \sim 0.05^{2} \bar{\Gamma}$. Note that this value is obtained without even considering the possibility of actively compensating for the spread in the couplings by suitably adjusting the detuning. This hints that under presently available optimal experimental conditions, the infidelity due to parameter spread becomes visible only long after the system has been severely decohered anyway. However, one can always intentionally choose parameters such that Eq. (18) is fulfilled and the full dynamics discussed here can be experimentally observed, including the ultimate measurement-induced decay of the temporarily produced entangled state.

The main challenging step to be taken experimentally before realizing this scheme in the laboratory is to operate in the single-shot qubit readout limit. This has been demonstrated very recently by the Saclay group using a Josephson bifurcation amplifier setup [58].

\section{CONCLUSIONS}

We have analyzed a very general experimentally directly relevant way to generate entangled multiqubit states using a dispersive phase-shift measurement of the collective state of several qubits inside a cavity. We have given criteria for the possibility to synthesize a given target state and studied the most relevant examples of Bell states as well as $W$ and GHZ states for two and three qubits. We have also discussed, and analyzed by extensive numerical simulations, the two major sources of imperfections in this setup, namely, decoherence and parameter spread. Finally, we have compared with presently reachable experimental parameters and conclude that this scheme could soon be tested in the laboratory.

Note added in proof. Recently, similar results concerning entanglement by measurement in circuit QED have been obtained in Ref. [59]

\section{ACKNOWLEDGMENTS}

We thank Jens Siewert for enlightening discussions. Support from the SFB 631, NIM, and the Emmy-Noether program (F.M.) of the DFG, as well as EuroSQIP, is gratefully acknowledged.
[1] F. Marquardt and C. Bruder, Phys. Rev. B 63, 054514 (2001).

[2] O. Buisson and F. Hekking, Macroscopic Quantum Coherence and Quantum Computing (Kluwer, New York, 2001).

[3] J. Q. You and F. Nori, Phys. Rev. B 68, 064509 (2003).

[4] A. Blais, R. S. Huang, A. Wallraff, S. M. Girvin, and R. J. Schoelkopf, Phys. Rev. A 69, 062320 (2004).

[5] A. Wallraff et al., Nature (London) 431, 162 (2004).

[6] H. Mabuchi and A. C. Doherty, Science 298, 1372 (2002).

[7] I. Chiorescu et al., Nature (London) 431, 159 (2004).

[8] J. Johansson, S. Saito, T. Meno, H. Nakano, M. Ueda, K. Semba, and H. Takayanagi, Phys. Rev. Lett. 96, 127006 (2006).

[9] D. I. Schuster, A. Wallraff, A. Blais, L. Frunzio, R. S. Huang, J. Majer, S. M. Girvin, and R. J. Schoelkopf, Phys. Rev. Lett. 94, 123602 (2005).
[10] A. Wallraff, D. I. Schuster, A. Blais, L. Frunzio, J. Majer, M. H. Devoret, S. M. Girvin, and R. J. Schoelkopf, Phys. Rev. Lett. 95, 060501 (2005).

[11] A. A. Houck, D. Schuster, J. Gambetta, J. Schreier, B. Johnson, J. Chow, J. Majer, L. Frunzio, M. Devoret, S. Girvin, and R. Schoelkopf, Nature (London) 449, 328 (2007).

[12] M. Hofheinz, E. Weig, M. Ansmann, R. Bialczak, E. Lucero, M. Neeley, H. Wang, J. Martinis, and A. Cleland, Nature (London) 454, 310 (2008).

[13] H. Wang, M. Hofheinz, M. Ansmann, R. Bialczak, E. Lucero, M. Neeley, A. Oconnell, D. Sank, J. Wenner, A. Cleland, and J. Martinis, e-print arXiv:0808.3279.

[14] J. Majer et al., Nat. Phys. 449, 443 (2007).

[15] M. Sillanpaa, J. Park, and R. Simmonds, Nature (London) 449, 438 (2007). 
[16] J. Fink, R. Bianchetti, M. Baur, M. Göppl, L. Steffen, S. Filipp, P. Leek, A. Blais, and A. Wallraff, e-print arXiv:0812.2651.

[17] P. Leek, J. Fink, A. Blais, R. Bianchetti, M. Goppl, J. Gambetta, D. Schuster, L. Frunzio, R. Schoelkopf, and A. Wallraff, Science 318, 1889 (2007).

[18] D. I. Schuster et al., Nature (London) 445, 515 (2007).

[19] F. Helmer, M. Mariantoni, E. Solano, and F. Marquardt, Phys. Rev. A 79, 052115 (2009)

[20] V. B. Braginsky, I. I. Vorontsov, and K. S. Thorne, Science 209, 547 (1980).

[21] V. B. Braginsky and F. Y. Khalili, Quantum Measurement (Cambridge University Press, Cambridge, 1992).

[22] M. Sarovar, H. S. Goan, T. P. Spiller, and G. J. Milburn, Phys. Rev. A 72, 062327 (2005).

[23] C. L. Hutchison, J. M. Gambetta, A. Blais, and F. K. Wilhelm, e-print arXiv:0812.0218.

[24] D. Rodrigues, C. Jarvis, B. Gyorffy, T. Spiller, and J. Annett, J. Phys.: Condens. Matter 20, 075211 (2008).

[25] J. Siewert and R. Fazio, J. Mod. Opt. 49, 1245 (2002).

[26] J. Lantz, M. Wallquist, V. S. Shumeiko, and G. Wendin, Phys. Rev. B 70, 140507(R) (2004).

[27] M. Wallquist, J. Lantz, V. Shumeiko, and G. Wendin, New J. Phys. 7, 178 (2005).

[28] L. F. Wei, Y. X. Liu, and F. Nori, Phys. Rev. Lett. 96, 246803 (2006).

[29] M. Wallquist, V. Shumeiko, and G. Wendin, e-print arXiv:cond-mat/0608209.

[30] M. Steffen, M. Ansmann, R. Bialczak, N. Katz, E. Lucero, R. McDermott, M. Neeley, E. Weig, A. Cleland, and J. Martinis, Science 313, 1423 (2006).

[31] K. Maruyama, T. Iitaka, and F. Nori, Phys. Rev. A 75, 012325 (2007).

[32] J. Q. You, X. B. Wang, T. Tanamoto, and F. Nori, Phys. Rev. A 75, 052319 (2007).

[33] J. Majer et al., Nature (London) 449, 443 (2007).

[34] A. Barchielli, Phys. Rev. A 34, 1642 (1986).

[35] W. Nagourney, J. Sandberg, and H. Dehmelt, Phys. Rev. Lett. 56, 2797 (1986).

[36] M. Porrati and S. Putterman, Phys. Rev. A 36, 929 (1987).

[37] H. Carmichael, An Open Systems Approach to Quantum Op- tics, Springer Lecture Notes Monographs Series No. 18 (Springer-Verlag, Berlin, 1993).

[38] J. I. Cirac, R. Blatt, A. S. Parkins, and P. Zoller, Phys. Rev. Lett. 70, 762 (1993).

[39] D. F. Walls and G. J. Milburn, Quantum Optics (Springer, New York, 1995).

[40] M. B. Plenio and P. L. Knight, Rev. Mod. Phys. 70, 101 (1998).

[41] S. Peil and G. Gabrielse, Phys. Rev. Lett. 83, 1287 (1999).

[42] C. W. Gardiner and P. Zoller, Quantum Noise (Springer-Verlag, Berlin, 2004).

[43] D. H. Santamore, A. C. Doherty, and M. C. Cross, Phys. Rev. B 70, 144301 (2004).

[44] S. Gleyzes, S. Kuhr, C. Guerlin, J. Bernu, S. Deléglise, U. Busk Hoff, M. Brune, J.-M. Raimond, and S. Haroche, Nature (London) 446, 297 (2007).

[45] J. Gambetta, A. Blais, M. Boissonneault, A. A. Houck, D. I. Schuster, and S. M. Girvin, Phys. Rev. A 77, 012112 (2008).

[46] H. Goan and G. Milburn, Phys. Rev. B 64, 235307 (2001).

[47] A. Scott and G. Milburn, Phys. Rev. A 63, 042101 (2001).

[48] K. Jacobs, Phys. Rev. A 67, 030301(R) (2003).

[49] C. Hill and J. Ralph, New J. Phys. 9, 151 (2007).

[50] D. Steck, K. Jacobs, H. Mabuchi, S. Habib, and T. Bhattacharya, Phys. Rev. A 74, 012322 (2006).

[51] H. M. Wiseman, Ph.D. thesis, University of Queensland, 1994.

[52] G. J. Milburn, Phys. Rev. A 44, 5401 (1991).

[53] A. A. Houck et al., Nature (London) 449, 328 (2007).

[54] B. Misra and E. C. G. Sudarshan, J. Math. Phys. 18, 756 (1976).

[55] F. Helmer, M. Mariantoni, A. Fowler, J. von Delft, E. Solano, and F. Marquardt, EPL 85, 50007 (2009).

[56] M. Mariantoni, M. Storcz, F. Wilhelm, W. Oliver, A. Emmert, A. Marx, R. Gross, H. Christ, and E. Solano, e-print arXiv:cond-mat/0509737.

[57] F. Marquardt, Phys. Rev. B 76, 205416 (2007).

[58] F. Mallet et al. (private communication).

[59] L. S. Bishop, L. Tornberg, D. Price, E. Ginossar, A. Nunnemkamp, A. A. Houck, J. M. Gambetta, J. Koch, G. Johansson, S. M. Girvin, and R. J. Schoelkopf, e-print arXiv:0902.0324. 